\title{
Precios del suelo y centros comerciales, un análisis de la ciudad de Bogotá
}

\author{
Julián Alberto Gutiérrez López ${ }^{1}$ \\ David Felipe Gómez Pimienta ${ }^{2}$ \\ Mallorie Joanna Uribe Cruz ${ }^{3}$ \\ Juan Camilo Moreno Ramírez ${ }^{4}$
}

Recibido: 04 de junio de 2019

Aprobado: 18 de julio de 2019

Gutiérrez, J. A., Gómez, D. F., Uribe, M. J., y Moreno, J. C. (2019). Precios del suelo y centros comerciales, un análisis de la ciudad de Bogotá. Revista Activos, 17(2), 17-43. DoI: https://doi.org/10.15332/25005278/5731

1 Economista de la Escuela de Ingenieros Julio Garavito, Magíster en Ciencias Económicas de la Universidad Santo Tomás. Profesor investigador de la Universidad El Bosque, Bogotá, Colombia. Correo: jagutierrezl@unbosque.edu.co ORCID: https://orcid.org/0000-0001-5296-0853.

2 Estudiante en la Facultad de Ciencias Económicas y Administrativas, Universidad El Bosque, Bogotá Correo: dfgomezp@unbosque.edu.co ORCID: https://orcid.org/0000-0002-1385-6014.

3 Estudiante en la Facultad de Ciencias Económicas y Administrativas, Universidad El Bosque, Bogotá Correo: mjuribe@unbosque.edu.co ORCID: https://orcid.org/0000-0002-7794-5833.

4 Contador Público de la Universidad Nacional de Colombia y especialista en Gerencia Financiera de la Universidad Jorge Tadeo Lozano. Es experto en IFrs de la firma Grant Thornton. Certificado por el ACCA en presentación de información financiera internacional. Consultor y asesor para entidades del Gobierno del Distrito Capital. Correo: juan.c.moreno@hotmail.com ORCID: https://orcid.org/0000-0002-2485-9050. 


\title{
Clasificación JEL: P25, M49
}

\section{Resumen}

El presente trabajo aborda el diseño y la aplicación de un modelo de precios hedónicos, mediante el cual se establecerá la elasticidad en el precio del metro cuadrado de terreno a la distancia a un centro comercial en la ciudad de Bogotá y a los demás factores socioeconómicos y de localización que puedan intervenir en la formación de dicho precio para el 2018. Para esto, se usó la información cartográfica de la totalidad de manzanas de la ciudad de Bogotá y todos los centros comerciales existentes a la fecha. Para el diseño del modelo econométrico, se tomó la información de la base de datos de la página de Infraestructura de Datos Espaciales para el Distrito Capital (IDECA), información socioeconómica suministrada por la Secretaría de Planeación Distrital y el inventario del Sistema Vial del Instituto de Desarrollo Urbano (IDU). Los resultados muestran una relación inversa entre la distancia a los centros comerciales y los precios del suelo, indicando que, a mayor distancia entre un predio y un centro comercial, el precio del suelo de dicho predio es más bajo, es decir que, al aumentar la distancia un $1 \%$, el valor del suelo caerá en $-0,28 \%$.

Palabras clave: precios hedónicos, centros comerciales, modelos de precios del suelo, modelo Von Thünen.

\section{Land Prices and Shopping Centers, an Analysis in the City of Bogota}

\begin{abstract}
This paper addresses the design and application of a hedonic pricing model, through which it will be stablished the price elasticity of the square meter of land in relation to its distance from a shopping center in the city of Bogota and other socioeconomic and location factors that may intervene in the
\end{abstract}


calculation of such price by 2018. To this end, cartographic information of all the blocks of the city of Bogota and all the existing shopping centers was used. For the design of the econometric model, information was taken from the database on the webpage of the Spatial Data Infrastructure for the Capital District (IDECA), the socioeconomic data provided by the Urban Development Institute, and the inventory of the Urban Development Institute (IDU). The results show an inverse relationship between the distance to shopping centers and land prices, indicating that the greater the distance between a property and a shopping center, the lower the price of the land in that property, ie, if the distance increases by $1 \%$, the value of the land decreases by $-0,28 \%$.

Keywords: hedonic prices, shopping centers, land prices models, Von Thünen model.

\section{Introducción}

Desde sus inicios en el bazar oriental, el foro romano y otras grandes construcciones europeas un sin número de galerías comerciales trataban de generar un aforo constante a sus vitrinas, los centros comerciales han estado en constante crecimiento no solo en términos de magnitud, sino en su relevancia dentro del día a día de los ciudadanos, ya sea convirtiéndose en referentes arquitectónicos, nodos de desarrollo urbano o simplemente como ejes de entretenimiento y comercio.

Dicha tendencia ha logrado a lo largo de los años un crecimiento considerable en su cantidad, entre los cuales cabe destacar los años ochenta y noventa donde la cifra se duplico en América Latina y que sirve de antesala para destacar la tendencia del 2000 a la fecha, donde el crecimiento fue tal, que el metraje se triplico. Esta tendencia fue jalonada por países como Brasil y México, sin embargo, en Colombia, a pesar de empezar un poco tarde en términos históricos, su ritmo ha sido de los más dinámicos en los últimos años, resaltando el comportamiento de la ciudad de Bogotá. 


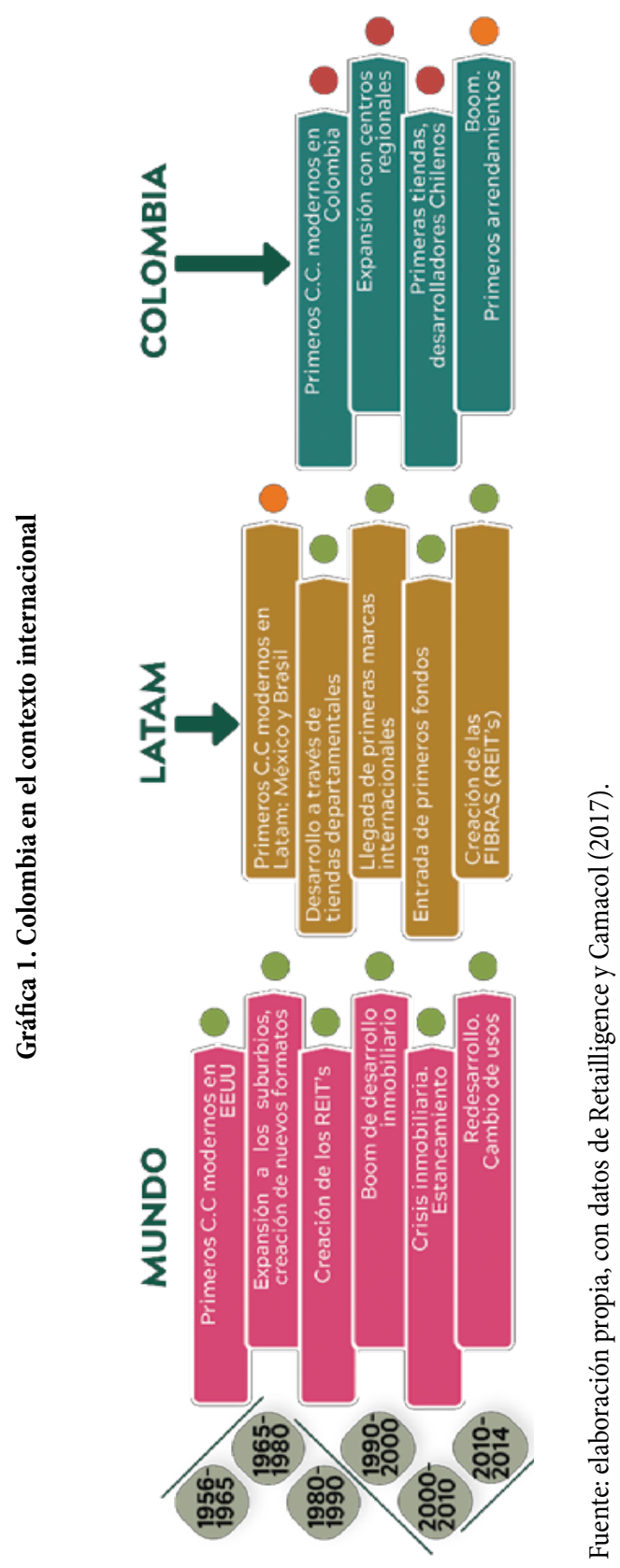


Pero dicha dinámica debe ser analizada detenidamente, ya que es la puerta para analizar cuáles son los potenciales que se pueden generar acorde a su distribución inicial. Las primeras unidades que se desarrollaron en la ciudad fueron en los años setenta (Unicentro y Centro 93) y se dieron en la zona residencial norte de ambos lados de la calle 100, dejando a Unicentro como el límite de desarrollo de los estratos altos de la ciudad. Ya en los ochenta se realiza la construcción de siete nuevos centros comerciales (Cedritos, Hacienda Santa Bárbara, Bulevar, Granahorrar, Metrópolis, Galerías y Floresta) impulsados por el crecimiento residencial y demográfico hacia el occidente de la ciudad (Retailligence y Camacol, 2017).

En los años noventa, dicha dinámica muta considerablemente, aunque no disminuyo su ritmo ya que se alcanzó un total de veintidós centros comerciales. Esta mutación estuvo enmarcada por el desarrollo periférico que sufrió la ciudad debido a los precios del suelo y a una transformación en el concepto de oferta y se empezaron a generar centros comerciales en zonas de ingreso medio bajo y bajo, tales como Bima, Unisur, entre otros (Retailligence y Camacol, 2017).

En la actualidad existen 56 centros comerciales para un total de más de millón ochocientos metros cuadrados donde se afianzo la tendencia del crecimiento periférico (Autopista Norte, Occidente, Américas y el sur de la Ciudad) y la consolidación de ciertas zonas donde su posición, sus procesos de renovación y su crecimiento demográfico generaron nuevos focos comerciales (Retailligence y Camacol, 2017).

\section{Revisión de la literatura}

Se mostrará cómo a lo largo de la historia se han generado estos procesos con el fin de lograr un mayor entendimiento en lo referente a la valorización del suelo, no solo en Bogotá, sino en diferentes ciudades y la aplicación de modelos de precios hedónicos, en cuanto a los centros comerciales y su influencia en el precio de la vivienda en la ciudad; además, con el fin de comprender más a fondo la aplicación del modelo mencionado anteriormente. 
En cuanto a la valoración del suelo, Borrero y Durán (1980), en su estudio titulado "El valor del suelo urbano y sus implicaciones en el desarrollo de la ciudad: análisis del caso de Bogotá", analizó el comportamiento de los precios de la tierra en la ciudad entre 1959-1978, estudio que fue actualizado en el año 1988, en donde se llegó a la conclusión que la tierra vale de acuerdo con el nivel social que la ocupe y en la medida que los niveles medios se acerquen al estrato alto, la tierra se valorizará.

Además, una investigación por parte de Doherty (2004) llamada: Funding Public Transport Development through Land Value Capture Programs, presenta varios ejemplos en Australia y otros países, analizando las obras de infraestructura pública y su influencia en transformaciones a usos mixtos y a densidades más altas; además, se determinan metodologías para definir los cambios del valor y se llegó a la conclusión que los métodos aplicados al finalizar las obras públicas son más contundentes que las proyecciones hechas antes de iniciarse los proyectos.

En el año 2008, se realizó un estudio por parte de la Lonja de Propiedad Raíz de Bogotá mediante el cual se analizaron 103 zonas de la ciudad en cuanto a sus usos comerciales, de servicios, industriales, residenciales, $y$ también por su ubicación, estrato, normatividad urbanística y antigüedad de desarrollo y consolidación, con el fin de establecer el valor definitivo para cada zona (Nigrinis, 2008). Después de realizar el análisis se concluyó que Bogotá es una ciudad pluricéntrica, en cuanto a su vivienda es monocéntrica, pues según el gráfico de Meyer el cono de alta renta o estratos altos se encuentra en la zona norte y así se va mezclando con los estratos medios y medio-bajos.

En esta misma línea de trabajo, se realizó la investigación El estudio del valor del suelo urbano en Cali (2012), donde se toman las cinco piezas urbanas que dividen la ciudad en el Plan de Ordenamiento Territorial del Municipio de Santiago de Cali y en base a estas se realiza la asignación de los valores comerciales unitarios teniendo en cuenta que los factores que influyen en este proceso son la altura, los índices de construcción, los aislamientos, entre otros. 
El estudio llevado a cabo por Gaitán Álvarez (2013), denominado "Incidence of the Agglomeration Economies in the Real Sector Located in the Urban Area of Bogotá", también muestra un análisis en cuanto a localización y concentración geográfica de diecinueve sectores reales de la economía de la ciudad basándose en la distancia de la localización entre firmas a través de la función K de Ripley; en donde se llega a la conclusión que los sectores de la economía capitalina muestran patrones de concentración específicos y se benefician de manera diversa de las economías de aglomeración.

Otro proyecto que analiza la variación en cuanto a la valorización del suelo se titula Estudio del valor del suelo de la ciudad de Bogotá, aplicativo web geográfico, estudio de caso localidad Antonio Nariño; mediante el cual se estudia el valor del suelo para la localidad Antonio Nariño en el marco del estudio del valor del suelo de la ciudad de Bogotá y se extiende el aplicativo web geográfico para consulta por los diferentes actores interesados (Molina y Bartolo, 2015). En esta investigación se llegó a la conclusión que la demanda del suelo ha aumentado por el incremento en la densidad poblacional.

En cuanto a la Formación de los precios del suelo urbano, Borrero (2016) analiza el desarrollo de la economía, los aspectos determinantes de los precios del suelo, su valorización y los ciclos de valor aplicables en América Latina. Se llegó, aquí, a la conclusión que se presenta una discriminación por usos y estratos sociales en las cuatro décadas estudiadas en Bogotá, además, las zonas centrales de comercio y negocios experimentaron una mayor depresión o menor valorización en cada década, en comparación con los estratos altos y las zonas industriales.

De igual manera, es pertinente especificar algunos estudios en los cuales se aplica el modelo de precios hedónicos y otras metodologías con el fin de establecer el valor del suelo de manera cuantitativa al tener en cuenta la cercanía que existe entre una vivienda y un centro comercial. Además, se pueden evidenciar estudios en los cuales se implementa el mismo modelo, pero en relación con las estaciones de Transmilenio. 
Principalmente, se encontró un estudio por parte de Santana y Núñez (2011), llamado: "Una aproximación hedónica al efecto de las preferencias por segregación en el precio del suelo urbano en Bogotá", mediante el cual se distingue la segregación socioeconómica como una variable significativa en la determinación del valor del suelo urbano residencial, empleando la distancia a estratos socioeconómicos; por otro lado, se concluye que la disponibilidad a pagar de los compradores de una vivienda es menor si se encuentra cerca de un asentamiento residencial de estratos bajos y es mayor si se encuentran cerca a los estratos cuatro y seis, lo que demuestra que este impacto en la distancia es desigual.

En cuanto a centros comerciales, Gonzáles y Montenegro (2008) llevaron a cabo el estudio: Impacto socioeconómico de la obra civil "Centro Comercial Santafe", en la comunidad adyacente correspondiente a los barrios de La Academia, La Uribe, El Verbenal y San José de Bavaria, en las localidades de Suba y Usaquén de la ciudad de Bogotá, D. C., que tiene como finalidad investigar el área de influencia directa e indirecta y el impacto que se generó durante la planeación, ejecución y puesta en servicio de la obra. La principal conclusión es que el impacto que tiene el Centro Comercial Santafé sobre la zona que se estudio fue positivo, pues la calidad de vida aumentó y su crecimiento está en constante desarrollo, y la valorización de los sectores cercanos experimenta una valorización a corto plazo.

Según Palacios (2015) mediante el estudio: Impacto socio espacial de la implantación de centros comerciales en Bogotá D. C., analiza cómo este factor es cada vez más protagónico en cuanto a la configuración de la estructura urbana de la ciudad y se identifican los factores que inciden en la fragmentación urbana y la segregación social. En donde se concluyó que los centros comerciales han contribuido a la separación de los grupos sociales, y se evidencia la diferencia de precios de acuerdo con el estrato social.

En esta misma línea de trabajo, García (2015), en su estudio: Impacto en el valor del suelo y cambios de uso por la implantación del Centro Comercial Centro Mayor, en el sector de Villa Mayor de la ciudad de Bogotá, realiza un análisis de variaciones en la dinámica inmobiliaria de un sector como 
resultado de la implantación de un centro comercial; se evidencia que algunas cifras se encuentran por debajo de las valorizaciones alcanzadas por los cuatro sectores estudiados cercanos al Centro Comercial Centro Mayor.

Otro estudio realizado en cuando a la presencia de centros comerciales, lo realizan Aguiar y Perdomo (2017) y se titula: Efectos de la presencia de centros comerciales sobre el valor del suelo en Bogotá; proyecto mediante el cual se explica la teoría económica de la renta del suelo, los costos de transporte, las aglomeraciones urbanas y sus externalidades asociadas, que se relaciona con la formación de las ciudades y su estructura urbana; además, se analiza la incidencia de los centros comerciales en el valor del suelo de la ciudad de Bogotá. Mediante este estudio se pudo concluir que el valor del suelo se obtiene en la medida que las personas buscan alojarse lo más cerca posible a las zonas en las que puedan obtener beneficios como productores o consumidores, es decir, que el valor del suelo está relacionado con la distancia y los costos de transportes asociados.

Como se mencionó anteriormente, en la investigación de este proyecto también se tienen en cuenta estudios previos en los cuales se aplica el modelo de precios hedónicos como herramienta para analizar los factores que influyen en el precio de una vivienda en relación con su cercanía a una estación de Transmilenio.

Mendieta y Perdomo (2007) realizaron un proyecto denominado: "Especificación y estimación de un modelo de precios hedónico espacial para evaluar el impacto de Transmilenio sobre el valor de la propiedad en Bogotá", en donde se incluye la variable distancia entre la propiedad y la estación más cercana al sistema de transporte masivo Transmilenio en Bogotá, Colombia. Aquí se explican las variaciones en el precio de la tierra en cuanto a variables de la vivienda, atributos ambientales de entorno, del vecindario, de seguridad y la distancia que ya fue mencionada, mediante un modelo espacial, en el cual se establece la relación de dicha distancia con el valor catastral. 
Cabe resaltar que (Perdomo, 2010), a través de la implementación de los precios hedónicos espaciales y la metodología de Propensity Score Matching, se estableció el cambio en el valor de las viviendas de la ciudad de Bogotá en cuanto a su cercanía a una estación de Transmilenio en la localidad de Suba y la Primera de Mayo con Boyacá, en donde se evidencia que las viviendas que tienen fácil acceso a una estación de Transmilenio muestran un diferencial de precios si se compara con las viviendas que su acceso al sistema es limitado.

Por último, Gutiérrez y Díaz (2017) en su tesis titulada Precios del suelo e infraestructura de transporte: caso aplicado al Sistema de Transmilenio en Bogotá, se determina la elasticidad en el precio según la distancia que existe a las estaciones de Transmilenio, mediante un modelo de precios hedónicos teniendo en cuenta variables tanto estructurales como de localización y socioeconómicas. Los resultados que arroja la investigación son:

Una relación inversa entre la distancia a las estaciones de Transmilenio y los precios del suelo, indicando que el precio del suelo de un "territorio" es más bajo a mayor distancia entre este y una estación. Desagregando el modelo a nivel socioeconómico se encuentra un mayor impacto para los estratos uno y dos con $15,5 \%$ y $6 \%$ respectivamente. Por otro lado, el efecto generado por áreas de influencia es más significativo para las distancias entre 200 y 500 metros, con un impacto de 4,4\%; lo que indica que, los agentes optan por localizaciones cercanas, pero no inmediatas a una estación de Transmilenio (Gutiérrez y Díaz, 2017b).

\section{Marco teórico}

\section{Orden urbano espacial}

Para poder identificar cómo se constituye el orden urbano espacial es necesario remitirse a los discursos de las ciencias sociales, los cuales recalcan que existen algunas regularidades según el contexto no aleatorio que tiene 
la configuración de la estructura intraurbana. Según Abramo (2001), es fundamental estudiar la evolución teórica que se relaciona con el modelo de ordenamiento intraurbano mediante las representaciones sobre el comportamiento de los hombres y los rasgos disciplinarios de las ciencias sociales con el fin de conocer cómo está constituido un orden espacial urbano.

El pensamiento económico a través de su existencia ha considerado el espacio como un componente esencial en cuanto a las decisiones de los individuos. Algunos autores como "Cantillon, Smith y Ricardo ya consideraban la importancia de la mano de obra y su relación con el espacio, sus consecuencias en temas productivos y la reciprocidad entre el campo y la ciudad" (Becerra, 2010, p. 9).

En un estudio que realizo Ricardo (2003) sobre la renta de la tierra y más específicamente sobre las "leyes de granos", explica que la renta diferencial "surge cuando se cultivan porciones de tierra con una fertilidad decreciente o debido a una localización diferencial” (Becerra, 2010, p. 9).

Por otro lado, las doctrinas de Von Thünen sobre la localización óptima de los cultivos agrícolas las cuales fueron aplicadas a las disposiciones de localización industrial empezaron a verse influenciadas por autores como Wilhem Roscher y Albert Schaffle; gracias a sus aportes se empezó a establecer un progreso de las ideas sobre la localización agrícola enfocada hacia una apreciación más urbana e industrial (Muiños, 2001).

Además, según García (1976) el trabajo de Von Thünen ha sido considerado como uno de los primeros aportes a la teoría de la localización, debido a que es el autor del primer modelo de orden espacial racional para la producción agrícola:

En una agricultura de subsistencia o autártica no queda diferenciado el espacio agrícola; pero con el paso a una agricultura de mercado o comercial, condicionada por la existencia de un mercado urbano, se desemboca en una diferenciación territorial del espacio agrícola. El modelo de Von Thünen estudia, de hecho, esta transformación espacial provocada por la 
sustitución de un sistema agrícola cerrado o autárquico por otro abierto o de mercado. (García, 1976, p. 12)

En el trabajo realizado por Alonso (1960), el autor se refiere al diseño metodológico de Von Thünen como un modelo que nace de una disposición urbana de una ciudad monocéntrica (único gran centro); en el cual se basan en la relación existente entre precio-distancia al centro y explican que si hay localizaciones distintas estas implican el desarrollo de ofertas de rentas diferentes para los propietarios del suelo.

En cuanto a esto, se concluye que "la renta ofrecida es función de la distancia al centro y de las ofertas urbanas de bienes públicos cuando se refiere al ámbito poblacional, esto se dispone con base al diferencial de ventas y costos de producción" (Gutiérrez y Díaz, 2017, p. 20). Por lo cual, los beneficios de una localización en específico se definen mediante la siguiente ecuación:

$$
\pi i(r, o u)=\varphi i(r, o u)-R i(r)
$$

En donde

si el marco institucional de operación de los mercados es de competencia en el desarrollo de actividades, los beneficios de los productores, $\pi i$, se establecen en términos de las tenciones entre promotores, $\varphi i$, y propietarios del suelo, Ri(r), de forma que la concurrencia obliga la cesión de todo excedente por encima de la sumatoria del costo de usos de los factores al propietario del suelo. (Gutiérrez y Díaz, 2017, p. 21)

Esto quiere decir que la renta de equilibrio implica lo siguiente:

$$
\text { R. }=\max \left\{\max \varphi_{i}(r, o u), 0\right\}=\max =\left\{\left(p_{i} q_{i}-c_{i}\right), 0\right\}
$$

Lo que quiere decir que la renta de equilibrio es la circundante de todas y cada una de las funciones de renta de cualquier uso y además de eso es la que propone cada producto inmobiliario que se ofrece; afirmación que se puede evidenciar a partir de la siguiente gráfica. 


\section{Gráfica 2. Modelo de Von Thünen}

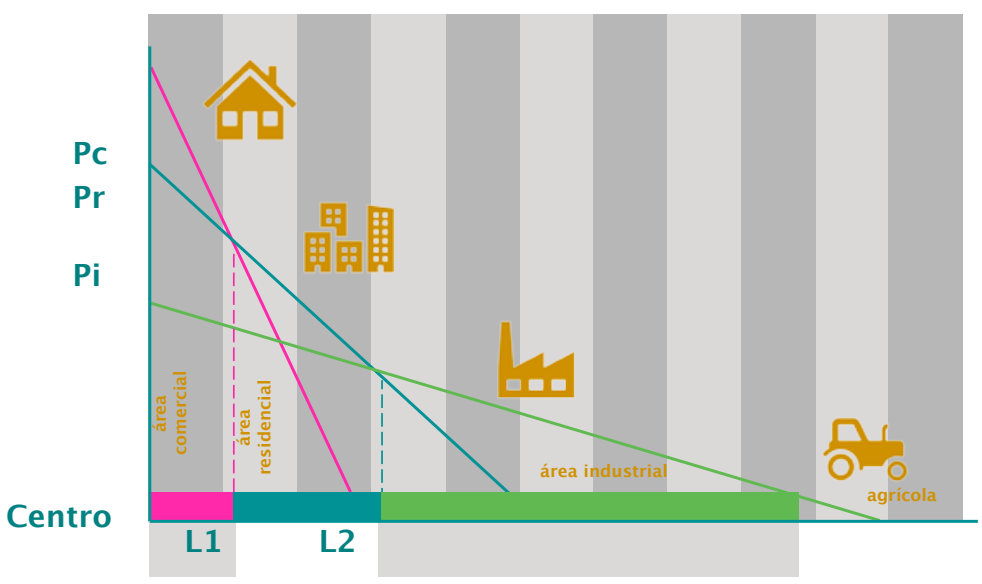

Fuente: elaboración propia, con datos de Abramo (2001).

En la gráfica 2 se puede apreciar que en el eje $y$ (ordenadas) se encuentra el valor de la renta, denominado $P$, y el eje $x$ (abscisas) representa la distancia al centro, denominada $D$. Mediante esta gráfica se pueden apreciar dos situaciones: por un lado, se puede evidenciar que en un lugar en específico de la cuidad, existe la posibilidad de brindar diferentes espacios edificados con distintas rentas del suelo; $y$, por otro lado, que se puede llevar a cabo al menos una actividad en cuanto respecta a los terrenos bajo los gradientes de renta que se señalan anteriormente. Sin embargo, hay que tener en cuenta que si se desarrollan las dos actividades que se relacionan tecnológicamente, estas ofrecerían la misma renta del suelo (Fujita y Thisse, 2002). Toda localización de empresas y hogares debe guiarse por la siguiente afirmación: el que ofrezca mayor renta del suelo es quien se queda con una localización (guiarse por la segunda fórmula).

Según la gráfica 2:

- $\varphi_{1}(\mathrm{r}, \mathrm{ou})=$ Se ubica cerca al centro, pues es la actividad económica que puede ofrecer una mayor renta del suelo y podrá generar más concentración de empleo. 
- $\varphi_{3}(\mathrm{r}, \mathrm{ou})=$ En este punto se presenta el caso contrario, debido a su lejanía del centro, su concentración de empleo será menor (Alonso, 1960).

Este último autor empieza concretando el inicio de la problemática que se presentan en cuanto a la distribución espacial de los funcionarios en la elección racional de la siguiente manera:

La distribución "geográfica" de los agentes en el espacio sería el resultado agregado de un conjunto de decisiones estrictamente individuales cuyo fin único sería maximizar una función de utilidad. De modo que el punto de partida de una teoría espacial que intentase definir la lógica de la distribución espacial según los principios de un agente maximizador racional (homo economicus) sería definir una teoría de la elección espacial de los individuos. (Abramo, 2001, p. 11)

De acuerdo con lo anterior, se definen los arbitrajes descentralizados que toman los agentes del mercado en cuanto a la estructura espacial, con el fin de definir la síntesis neoclásica de ordenamiento territorial (Gutiérrez y Díaz, 2017); esta, a su vez, será el punto base, en cuanto a localización, que se llevará a cabo en este proyecto por medio del modelo econométrico establecido.

Basándonos en lo que plantea Abramo (2001), el método que se implementará se basa en la maximización de la utilidad de un consumidor en donde la localización residencial será tomada en el grado de satisfacción que dichos bienes les pueden brindar. El consumo o canasta óptima se compondrá por dos tipos de bienes:

- Bienes y servicios que conforman la dimensión espacial, que a su vez se dividen en:

o El terreno ocupado por la familia, que se representa como $q$.

o La distancia de este al centro de la ciudad, tomada como $t$. 
- No pertenecen a dicha dimensión y se representa como $z$, que es tomada como un compuesto (único bien calificado).

En conjunto estas variables representan la función de utilidad que se traduce en la siguiente ecuación:

$$
\mathbf{U}=\mathbf{U}(\mathbf{z}, \mathbf{q}, \mathbf{t})
$$

En cuanto a las curvas de indiferencia, se puede presentar una situación en la cual una de las anteriores variables nombradas es constante (Gutiérrez y Díaz, 2017), en este caso se podrían presentar tres alternativas o combinaciones diferentes:

- Consumo de espacio y bien compuesto.

- Consumo del bien compuesto y distancia al centro de negocios.

- Consumo de espacio y distancia.

Según esto, Abramo (2001) explica que:

si el individuo es racional, dado que los empleos y los bienes compuestos están disponibles en el CBD (centro de negocios), él siempre va a preferir vivir lo más cerca posible del centro de la ciudad, o sea, desde que el individuo privilegie las comodidades de acceso a esos bienes, su satisfacción será tanto más importante cuanto más próximo del centro él estuviera. Es lo mismo que decir que el aumento de la distancia entre el lugar de residencia y el centro de la ciudad tenderá a producir una utilidad negativa o descontento (insatisfacción). (Abramo, 2001, p. 15)

\section{Precios hedónicos}

La Teoría de Precios Hedónicos pretende explicar el valor de un bien raíz, entendido como un conjunto de atributos (superficie, aptitud de uso del suelo, calidad de la construcción, diseño interior y exterior, áreas verdes, 
ubicación, características del vecindario, etc.), en función de cada uno de ellos, obteniendo sus respectivas valoraciones y, por ende, demandas implícitas. (Lever, 2000, p. 12 )

Dicho esto, se entiende que en algunas situaciones este conjunto de atributos influye en el precio del bien, pero también interfieren las características que marcan la diferencia; estas son las que el consumidor valora, quien finalmente interviene en dicho precio.

Esta teoría tiene sus inicios en los estudios llevados a cabo por Ridker y Henning (1967), mediante los cuales se examinó el efecto y relación que tiene la contaminación del aire y el precio de mercado de las viviendas teniendo también en cuenta la influencia que tienen otros factores como lo son las características propias del inmueble y el vecindario donde se encuentra.

El vacío que se encontró se relaciona con la identificación del anterior supuesto, pero el proceso para cuantificarlo es un poco más complejo. Supongamos que una persona está dispuesta a pagar un mayor precio por un bien (vivienda) ubicado en un sector que tiene mayor cantidad de espacio público que por una que tenga las mismas características, pero que carece de espacio público. En este caso es difícil encontrar dos viviendas en donde solo se tome en cuenta el anterior criterio propuesto; así, es en este momento que se tiene que evaluar cada atributo por separado teniendo en cuenta la relación que existe entre estos.

Por otro lado, aunque sus inicios se contribuyen al año 1967, existen desacuerdos sobre esta afirmación pues para algunos autores esta teoría nace por Zvi Griliches que es el padre del enfoque hedónico moderno (Berndt, 1990).

Pero hay que tener en cuenta que existen otros autores que hablan sobre el tema como Andrew Court, del cual se dice utilizó el término hedonic en un estudio que realizó en el año 1939; esto se sabe gracias a Allen Goodman (1998) quien lo especifica en uno de sus estudios; este término 
se hizo popular varios años después gracias a las investigaciones realizadas por Rosen (1974).

En esta misma línea, actualmente se aplica el método de precios hedónicos en cuanto a factores relacionados con el medio ambiente y a su influencia en el precio de la vivienda; esto al tener en cuenta la cercanía que esta tenga a algún "parque forestal" que, al igual que en el caso de los centros comerciales, se busca cuantificar las diferentes variables para que en conjunto se analice la formación del respectivo precio.

\section{Método}

El tipo de esta investigación es aplicado, ya que, conforme a su objetivo, el ámbito de estudio se encuentra delimitado a la ciudad de Bogotá; de igual manera, según el nivel de profundización en el objeto de estudio, esta investigación es explicativa, ya que su fin es determinar las causas y consecuencias de un fenómeno concreto.

Conforme al método, su enfoque es correlacional, ya que determina la relación de las variables socioeconómicas, espaciales y, como principal variable de estudio, la distancia a los centros comerciales con respecto al valor del metro cuadrado del terreno. Para esto, el tipo de datos escogidos son variables cuantitativas de corte transversal para el año 2018, con las cuales se obtienen mayores grados de control e inferencia, dichas variables son: 
Tabla 1. Variables

\begin{tabular}{|c|c|c|}
\hline Variable & Descripción & $\begin{array}{l}\text { Signo } \\
\text { esperado }\end{array}$ \\
\hline 1_zhgvm2terr_ponde & $\begin{array}{l}\text { Corresponde al logaritmo natural del valor del } \\
\text { metro cuadrado de terreno ponderado por área } \\
\text { de cada predio en la manzana. }\end{array}$ & \\
\hline 1_dens_pob & $\begin{array}{l}\text { Corresponde al logaritmo natural del índice de } \\
\text { población de la manzana. }\end{array}$ & Negativo \\
\hline 1_ind_ser_pub & $\begin{array}{l}\text { Corresponde al logaritmo natural del } \\
\text { índice de servicios públicos de la manzana. }\end{array}$ & Positivo \\
\hline 1_ind_educa & $\begin{array}{l}\text { Corresponde al logaritmo educación de las } \\
\text { personas que conforman la manzana. }\end{array}$ & Positivo \\
\hline 1_dist_transmi7 & $\begin{array}{l}\text { Corresponde al logaritmo natural de la distancia } \\
\text { euclidiana mínima de la manzana a la boca de } \\
\text { una estación o una parada de Transmilenio. }\end{array}$ & Negativo \\
\hline 1_dist_centgs & $\begin{array}{l}\text { Corresponde al logaritmo natural de la distancia } \\
\text { euclidiana mínima de la manzana a una } \\
\text { centralidad de Bogotá calculada mediante la } \\
\text { metodología de Guilliano y Small. }\end{array}$ & Negativo \\
\hline 1_dist_vart & $\begin{array}{l}\text { Corresponde al logaritmo natural de la distancia } \\
\text { euclidiana mínima de la manzana a una vía } \\
\text { arterial. }\end{array}$ & Negativo \\
\hline 1_ave_pisos & $\begin{array}{l}\text { Corresponde al logaritmo natural del promedio } \\
\text { del número de pisos de la manzana. }\end{array}$ & Positivo \\
\hline 1_acp_sitios & $\begin{array}{l}\text { Corresponde al logaritmo natural del primer } \\
\text { componente de las distancias euclidianas míni- } \\
\text { mas de la manzana a cada uno de los puntos del } \\
\text { sitios de interés definidos por el IDECA. }\end{array}$ & Negativo \\
\hline Estrato & $\begin{array}{l}\text { Corresponde al estrato predominante de la } \\
\text { manzana. }\end{array}$ & Positivo \\
\hline 1_dis_cc & $\begin{array}{l}\text { Corresponde al logaritmo natural de la distancia } \\
\text { euclidiana mínima de la manzana a un centro } \\
\text { comercial. }\end{array}$ & Negativo \\
\hline
\end{tabular}

Fuente: cálculos propios. 


\section{Resultados}

Determinadas las variables a usar en el modelo de precios del suelo, se procedió a calcular un modelo general para toda la ciudad.

regl_zhgvm2terr_ponder l_dens_pob l_dens_emp l_ind_ser_pub l_ind_educa l_dist_transmi7 l_dist_centgs l_dist_vart l_ave_pisos l_acp_sitios l_estrato l_dis_cc, robust

Tabla 2. Modelo de precios del suelo

\section{Linear regression}

$\begin{array}{ll}\text { Number of obs } & =37,965 \\ \mathrm{~F}(11,37953) & =2548.13 \\ \text { Prob }>\mathrm{F} & =0.0000 \\ \text { R-squared } & =0.5267 \\ \text { Root MSE } & =.57029\end{array}$

\begin{tabular}{l|r|r|r|r|r|r}
\hline 1_zhgvm2terr_ $\mathbf{r}$ & Coef. & $\begin{array}{c}\text { Robust } \\
\text { Std. Err. }\end{array}$ & $\mathbf{t}$ & $\mathbf{P}>|\mathbf{t}|$ & $\begin{array}{c}\text { [95_\% } \\
\text { Conf. }\end{array}$ & Interval] \\
\hline 1_dens_pob & -.2014688 & .0094454 & -21.33 & 0.000 & -.219982 & -.1829556 \\
\hline 1_dens_emp & .0032394 & .0002629 & 12.32 & 0.000 & .0027241 & .0037546 \\
\hline 1_ind_ser_pub & .1621844 & .0175387 & 9.25 & 0.000 & .127808 & .1965608 \\
\hline 1_ind_educa & .0466563 & .0119374 & 3.91 & 0.000 & .0232586 & .0700539 \\
\hline 1_dist_transmi7 & -.0634772 & .0094392 & -6.72 & 0.000 & -.0819783 & -.044976 \\
\hline 1_dist_centgs & -.0011609 & .0002648 & -4.38 & 0.000 & -.0016798 & -.000642 \\
\hline 1_dist_vart & -.0024949 & .0015946 & -1.56 & 0.118 & -.0056204 & .0006306 \\
\hline 1_ave_pisos & .0069269 & .0012172 & 5.69 & 0.000 & .0045411 & .0093126 \\
\hline 1_acp_sitios & -.3947393 & .0136165 & -28.99 & 0.000 & -.4214279 & -.3680507 \\
\hline 1_estrato & .0266828 & .00087 & 30.67 & 0.000 & .0249776 & .0283881 \\
\hline 1_dis_cc & -.2841264 & .0050632 & -56.12 & 0.000 & -.2940503 & -.2742025 \\
\hline _cons & 19.29117 & .0919463 & 209.81 & 0.000 & 19.11095 & 19.47138 \\
\hline
\end{tabular}

Fuente: elaboración propia. 


\section{Discusión}

En el anterior modelo se evidencia que la elasticidad del precio con respecto a la densidad de la población presenta un signo negativo, este radica en las diferentes tasas de natalidad en los diferentes ingresos, haciendo así que las localizaciones de precios más bajos presenten mayor número de hijos; del mismo modo, podemos apreciar con las variables de densidad de empleo y la de las centralidades de la ciudad que conforme aumenta el empleo o disminuye la distancia hacia un centro de empleo aumenta o disminuye el precio del suelo respectivamente.

Con respecto al índice de servicios públicos y al promedio del número de pisos, podemos apreciar que las elasticidades tienen signos positivos, lo cual va acorde a la realidad mundial e implica que a mayor cobertura de servicios públicos y a mayor número de pisos mayor precio del suelo.

Con respecto a la variable del índice de educación, variable que se incluye como un proxy del ingreso, podemos apreciar que dicha elasticidad presenta un signo positivo, lo que indica, como se mencionó en el modelo de Von Thünen, que las personas de altos ingresos ofrecen mayores rentas del suelo, generando así mayores precios de mercado.

Con respecto a las variables de localización se debe destacar la variable de los sitios de interés, índice que agrupa la cercanía a equipamientos, sitios turísticos, colegios, universidades, entre otros, podemos evidenciar que dichas elasticidades presentan signo negativo, lo que indica que las manzanas más distantes de dichos puntos de atracción presentan menores precios del suelo.

En cuanto a la variable que nos concierne en esta investigación, distancia a los centros comerciales "1_dis_cc", encontramos que la elasticidad es de $-0,28 \%$, lo que indica que conforme la distancia incrementa en un $1 \%$ el precio del suelo disminuye en dicha proporción; esta elasticidad toma relevancia por la disputa que coexiste entre los atractivos para el sector residencial (mayor oferta de bienes y servicios) y los del sector comercial, 
los cuales tratan de generar economías de aglomeración alrededor del centro comercial.

\section{Aspectos financieros y económicos}

Bajo la dinámica contable, el impacto de la valoración de los predios tiene un efecto cuantitativo directo sobre la medición inicial y posterior en los estados financieros de su propietario o de una entidad. De este modo, bajo la premisa de la Norma Internacional de Información Financiera correspondiente a propiedades de inversión, dicho impacto en la valoración se conoce como un efecto de valor razonable sobre un bien que se posee con el fin de generar rentas o plusvalías. La IFRs Foundation (2015) señala que:

Las propiedades de inversión se tienen para obtener rentas, apreciación del capital o ambas. Por lo tanto, una propiedad de inversión genera flujos de efectivo que son en gran medida independientes de otros activos poseídos por la entidad. (p. 5)

La norma internacional considera que son ejemplos de propiedades de inversión los terrenos con fines de apreciación del capital en el largo plazo, puesto que no se tiene previsto su venta en un momento cercano determinado. Así mismo, se consideran ejemplos de propiedades de inversión los terrenos para un uso futuro no determinado, las edificaciones para alquilar bajo la modalidad de arrendamiento operativo, así como los inmuebles en proceso de construcción y que, una vez finalizado dicho proceso, se tenga previsto ponerlo en disposición para constituir un arrendamiento operativo.

Desde el punto de vista de la situación financiera de los propietarios o entidades con el derecho de uso sobre las propiedades de inversión, este se considera un activo, ya que es probable que genere beneficios económicos futuros que fluyan hacia el propietario o la entidad. Dicho valor debe contar con una medición fiable, esta medición se debe basar en estimados contables, los cuales permitan garantizar la razonabilidad de su valoración, tal como los desarrollados a lo largo del presente escrito. 
Ahora bien, este impacto es reflejado a través de la medición inicial y posterior de la propiedad al centrarse específicamente en la medición posterior que toma como referencia dos métodos de valoración: el valor razonable y el costo. Para efecto del presente estudio, la valoración preponderante corresponde a una medición a valor razonable. De este modo, la IFRS Foundation (2015) señala que:

Cuando se mide el valor razonable de una propiedad de inversión [...], una entidad se asegurará de que el valor razonable refleja, entre otras cosas, los ingresos por alquileres de los arrendamientos actuales y otros supuestos que los participantes del mercado utilizarían al fijar el precio de la propiedad de inversión en condiciones de mercado actuales. (p. 8)

Conforme a lo anterior, la norma hace referencia a que aquellos supuestos fijan los precios y son reconocidos como una medición fiable, tales como: la relación de variables socioeconómicas, espaciales y, como variable principal de estudio, la distancia a los centros comerciales y su impacto en el valor del metro cuadrado de los terrenos; estos corresponden a estimados contables que fijan un precio según las condiciones actuales en las que se encuentran los participantes del mercado.

En una medición de valor razonable para activos no financieros, tales como los inmuebles y terrenos, se deberá tener en cuenta la capacidad de los participantes del mercado para generar beneficios económicos futuros mediante el mejor y máximo uso del activo, lo cual implica aspectos tales como:

- Uso de acuerdo con las características físicas del activo.

- Uso de acuerdo con las restricciones legales.

- Uso bajo condiciones financieras factibles, es decir, su capacidad para generar rentabilidad teniendo en cuenta su espacio y sus restricciones legales. 
Finalmente, es importante señalar que las pérdidas y ganancias derivadas del cambio en este valor razonable, dadas las anteriores variables en las propiedades de inversión, tendrán efecto sobre el resultado del ejercicio (periodo) en las que surjan. Esto significa que, de acuerdo a las políticas contables y a los periodos de medición, este valor será actualizado como un aumento o disminución del activo, seguido de un aumento o disminución en el resultado del periodo sea como un ingreso o gasto.

\section{Conclusiones}

A lo largo de la historia del mercado inmobiliario bogotano se ha evidenciado la creación de centros comerciales y su influencia en los precios del suelo, esta dinámica aunque nace tarde, principalmente por un modelo de desarrollo en ventas y copropiedad por parte de los desarrolladores, al compararla con países como Brasil y México se caracteriza por su buen ritmo y su creciente evolución.

El ritmo de crecimiento del sector inmobiliario en dicho aspecto ha llevado a juzgar, a primera vista, que los mercados ya se encuentran saturados y que los precios del suelo no recogen los efectos de la cercanía a dichos focos; pero, al realizar un análisis cuantitativo y cualitativo se encuentra que dicha afirmación está más que errada, ya que el potencial de expansión está dado principalmente por dos motivos: primero, porque aún existen zonas de la ciudad sin una cobertura optima y, segundo, por el desarrollo residencial vertical de la ciudad, justificado en la alta elasticidad en el precio del metro cuadrado de la distancia a dichos centros comerciales. 


\section{Referencias}

Abramo, P. (2001). Mercado y orden urbano: del caos a la teoría de la localización. Rio de Janeiro: Bertrand Brasil.

Aguiar Osorio, J. J., y Perdomo Strauch, Á. (2017). Efectos de la presencia de centros comerciales sobre el valor del suelo en Bogotá. Bogotá: Escuela Colombiana de Ingeniería Julio Garavito.

Alonso, W. (1960). A Theory of the Urban Land Market. Papers and Proceedings Regional Science Association, 6, 149-157.

Araque, A., Gutiérrez, J. y Quenguan, L. (2017). El espacio público en la ciudad: una aproximación desde los precios hedónicos. Revista Sociedad y Economía, 33(2), 77-98.

Berndt, E. R. (1990). The Practice of Econometrics: Classic and Contemporary. Cambridge: Addison-Wesley Publishing Company

Berndt, E. R., Griliches, Z. y Rappaport, N. (1995). Econometric Estimates of Prices Indexes for Personal Computers in the 1990's. Journal of Econometrics, 68, 243-268.

Borrero Ochoa, O., y Durán de Gámez, E. (1980). El valor del suelo urbano y sus implicaciones en el desarrollo de la ciudad: análisis del caso de Bogotá. Bogotá: Centro Nacional de Estudios de la Construcción-CENAC.

Borrero, O. (2016). Formación de los precios del suelo urbano. Bogotá: Educación a Distancia-Programa para América Latina y el Caribe.

Cámara de Comercio de Cali. (2012). Estudio del valor del suelo urbano en Cali. Cali: Lonja de Propiedad Raíz de Cali y Valle del Cauca.

Doherty, M. (2004). Funding Public Transport Development through Land Value Capture Programs. Sydney: University of Technology, Institute for Sustainable Futures.

Fujita, M., (1989). Urban Economic Theory: Land Use and City Size. Cambridge: Cambridge University Press.

Fujita, M. y Thisse, J. (2002). Economics of Agglomeration. Cities, Industrial Location and Regional Growth. Cambridge: Cambridge University Press. Gaitán Álvarez, J. (2013). Incidence of the Aglomeration Economies in the Real Sector in the Urban Area of Bogotá. Ensayos sobre Politica Económica, 31(70), 157-214. 
García Galindo, W. (2015). Impacto en el valor del suelo y cambios de uso por la implantación del Centro Comercial Centro Mayor, en el sector de Villa Mayor de la ciudad de Bogotá. Bogotá: Unidad Administrativa Especial de Catastro Distrital - UAECD.

García, D. (1976). Valor actual del modelo de Von Thünen y dos comprobaciones empíricas. Revista de Geografía, 10, 11-33.

Goodman, Allen C. (1998). Andrew Court and the Invention of Hedonic Price Analysis. Journal of Urban Economics, 44(2), 291-298.

González Saavedra, R., y Montenegro López, J. D. (2008). Impacto socioeconómico de la obra civil "Centro Comercial Santafé", en la comunidad adyacente correspondiente a los barrios de La Academia, La Uribe, El Verbenal y San José de Bavaria, en las localidades de Suba y Usaquén de la ciudad de Bogotá, D.C. (Tesis de pregrado, Universidad de la Salle). Recuperado de http://repository.lasalle.edu.co/bitstream/handle/10185/15399/T40.08\%20G589i.pdf;jsessionid=9494A60250C0B9A 8800D16CC7AE59545? sequence $=1$

Gutiérrez López, J. A., y Díaz Jiménez, M. A. (2017). Precios del suelo e infraestructura de transporte: Caso aplicado al Sistema de Transmilenio en Bogotá (Tesis de maestría, Universidad Santo Tomás). Recuperado de https://repository.usta.edu.co/bitstream/handle/11634/9130/ GutierrezJulian2017.pdf?sequence $=1$ \&isAllowed $=\mathrm{y}$

IFRS Foundation. (2015). La Norma Internacional de Información Financiera 13 Medición del Valor Razonable (NIIF 13). Recuperado de http:// www.ctcp.gov.co/proyectos/contabilidad-e-informacion-financiera/ documentos-organismos-internacionales/compilacion-marcostecnicos-de-informacion-financi/1534363802-6328

IfRS Foundation, (2016). Norma Internacional de Contabilidad 40 Propiedades de Inversión. London. Recuperado de https://www.mef.gub. uy/10192/1/mef/norma-internacional-de-contabilidad-n-\%C2\%B040-nic-40.html.

Lever, G. (s. f.). El modelo de precios hedónicos. Recuperado de http://www. ucipfg.com/Repositorio/MAES/PED/Semana4/PreciosHedonicos.pdf Mendieta, J. C., y Perdomo, J. A. (2007). Especificación y estimación de un modelo de precios hedónico espacial para evaluar el impacto de 
Transmilenio sobre el valor de la propiedad en Bogotá. Documentos CEDE, 22, 1-44.

Molina Munevar, O. H., y Bartolo Moscoso, J. A. (2015). Estudio del valor del suelo de la ciudad de Bogotá, aplicativo web geográfico, estudio de caso localidad Antonio Nariño (Tesis de pregrado, Universidad Distrital Francisco José de Caldas). Recuperado de http://repository.udistrital.edu.co/bitstream/11349/2691/1/MOLINA\%20MUNEVAR\%20 Oscar\%20Hernan\%20BARTOLO\%20MOSCOSO\%20Julian\%20 Alejandro.pdf

Muiños, B. (2001). Territorio, movilidad de mano de obra y formación del mercado de trabajo. El pensamiento económico espacial hasta la II Guerra Mundial. Revista Electrónica de Geografía y Ciencias Sociales, $V(94), 84$.

Nigrinis, M. I. (2008). El valor del suelo urbano en Bogotá. Bogotá: Lonja de Propiedad Raíz de Bogotá.

Palacios, A. (2015). Impacto socio espacial de la implantación de centros comerciales en Bogotá, D.C. Perspectiva Geográfica, 20(2), 319-338.

Perdomo, J. A. (2010). Una propuesta metodológica para estimar cambios sobre el valor de la propiedad: estudio de caso para Bogotá aplicando Socre Matching y Precios Hedónicos Espaciales. Lecturas de Economía, 73, 49-65.

Retailligence y Camacol. (septiembre-octubre, 2017). Estudio de centros comerciales. Construnota, 67. Recuperado de https://construnota. camacolcundinamarca.co/edicion-67/84-gesti\%C3\%B3n-gremial/370estudio-de-centros-comerciales.html

Ricardo, D. (2003). Principios de economía política y tributación (P. De La Núez y C. Rodríguez, trads.). Madrid: Ediciones Pirámide.

Ridker, R. Y Henning, J. (1967) The determinants of residential property values with special reference to air pollution. The Review of Economics and Statistics, 4, 246-257.

Rosen, S. (1974). Hedonic Prices and Implicit Markets: Product Differentiation in Perfect Competition. Journal of Political Economy, 82(1), 34-55. 
Santana Viloria, L. y Núñez Camargo, L. (2011). Una aproximación hedónica al efecto de las preferencias por segregación en el precio del suelo urbano en Bogotá. Equidad y Desarrollo, 16, 139-162.

Valbuena, S., Castiblanco, C., y Palacios, M. T. (2009). Descripción de las metodologías existentes de valoración de compensaciones ambientales $y$ sociales que seas aplicables al contexto colombiano. Bogotá: Instituto de Investigación de Recursos Biológicos Alexander von Humboldt. 